\title{
Arritmias Fetais
}

\author{
Lilian Maria Lopes, Marcelo Zugaib
}

São Paulo, SP

O interesse nas arritmias cardíacas fetais tem aumentado nos últimos anos, conseqüente à maior utilização dos métodos de monitorização fetal ${ }^{1}$. Deixando de ter cunho exclusivamente acadêmico, trabalhos recentes demonstram a precisão diagnóstica das arritmias pela ecocardiografia (ECO), bem como os resultados animadores em termos de tratamento com antiarrítmicos ${ }^{2-5}$.

\section{Sistema de condução e fisiologia miocárdica}

O sistema de condução do coração fetal está funcionalmente maduro ao redor da $16^{\mathrm{a}}$ semana. O nó sinusal encontra sua própria artéria para irrigação na $10^{\mathrm{a}}$ semana e apresenta um pouco menos de colágeno, quando comparado ao nó sinusal de pessoa adulta. O nó atrioventricular (AV) é formado na $10^{\mathrm{a}}$ semana, separadamente do feixe de Hiss, sendo que a união de ambos é feita secundariamente.

As cardiopatias congênitas com defeitos septais deslocam o sistema de condução, porém, raramente o interrompem. O sistema de condução é composto pelos seguintes elementos: nó sinusal; vias preferenciais atriais; nó AV; bifurcação dos ramos do feixe de Hiss em direito e esquerdo; rede ou fibras de Purkinje; miocárdio ventricular.

A principal característica da fisiologia miocárdica, determinante dos mecanismos de arritmia, é a automaticidade, que deve ser entendida como propriedade apenas das fibras miocárdicas especializadas, que formam o sistema de condução e não do miocárdio contráctil, como um todo. Refere-se à origem rítmica espontânea dos impulsos que são conduzidos para todas as partes do coração.

O miocárdio contráctil, por sua vez, apresenta, como característica, os períodos refratários relativo e absoluto. $\mathrm{O}$ período refratário absoluto é quando a fibra miocárdica, uma vez contraída, apresenta um intervalo de tempo finito durante o qual não pode ser estimulada novamente. Ao final desse tempo, começa o período refratário relativo, quando volta a apresentar, porém não completa, resposta frente a um estímulo. O período refratário absoluto é que protege o coração das arritmias.

A arritmia resulta, portanto, de uma automaticidade anormal, uma condução anormal ou da combinação de ambos.

Hospital das Clínicas da Faculdade de Medicina da USP - São Paulo

Correspondência: Lilian Maria Lopes - Rua Batista Caetano, 79/111 - 04108-130

- São Paulo, SP

\section{Classificação}

A classificação das arritmias diagnosticadas pela ECO fetal será suscintamente comentada, uma vez que o significado das arritmias diagnosticadas intra-útero é, freqüentemente, diferente daquelas diagnosticadas no período neonatal ou posterior ${ }^{6}$.

Arritmias sinusais - Taquicardia sinusal - Freqüência cardíaca (FC) em torno de 180bpm, às vezes intermitente. Causas: hipóxia fetal, ansiedade materna, febre materna, ingestão materna de atropina, escopolamina ou isoxsuprina, infecção por citomegalovírus. Diagnóstico diferencial: taquicardia supraventricular.

Bradicardia sinusal - FC $\leq 100 \mathrm{bpm}$. Causas: compressão de cordão umbilical ou cabeça fetal, hipotensão e convulsão materna; bloqueio anestésico paracervical, administração materna de propranolol ou reserpina. Diagnóstico diferencial: bloqueio atrioventricular (BAV).

Bloqueio sino-atrial - Queda da FC $\leq 60 \mathrm{bpm}$, podendo ocorrer conseqüentemente, batimentos de escape. Causas: compressão importante da cabeça ou cordão umbilical, estímulo parassimpático, hiperpotassemia.

Arritmias supraventriculares - Extra-sistolia supraventricular - O diagnóstico é feito através da demonstração do batimento precoce seguido de pausa não compensatória pelo modo-M, passando o cursor pela parede atrial. Podem ocorrer a partir da $18^{\mathrm{a}}$ semana de gestação e representam $3 \%$ a $5 \%$ das arritmias que ocorrem durante o trabalho de parto ${ }^{7,8}$. Não são decorrentes de hipóxia e sua evolução é conhecidamente benigna, desaparecendo espontaneamente durante a $1^{\text {a }}$ semana de vida. Mesmo freqüentes, bigeminadas ou trigeminadas, não mudam a conduta no parto, que deve ser obstétrica e não indicam terapêutica antiarrítmica fetal. Uma forma interessante de extrasistolia atrial é quando as mesmas se apresentam bigeminadas ou trigeminadas e não conduzidas para o ventrículo por serem precoces e encontrarem o nó AV ainda em período refratário (fig. 1). Pela bradicardia fixa que provocam são freqüentemente referidas para ECO fetal, com a hipótese diagnóstica de BAV. Entretanto, todos os nossos casos evoluíram de forma benigna com desaparecimento das extrasístoles antes ou após o parto.

Taquicardia supraventricular - FC > 200bpm, com intervalos regulares entre os batimentos. Pode ser não susten- 


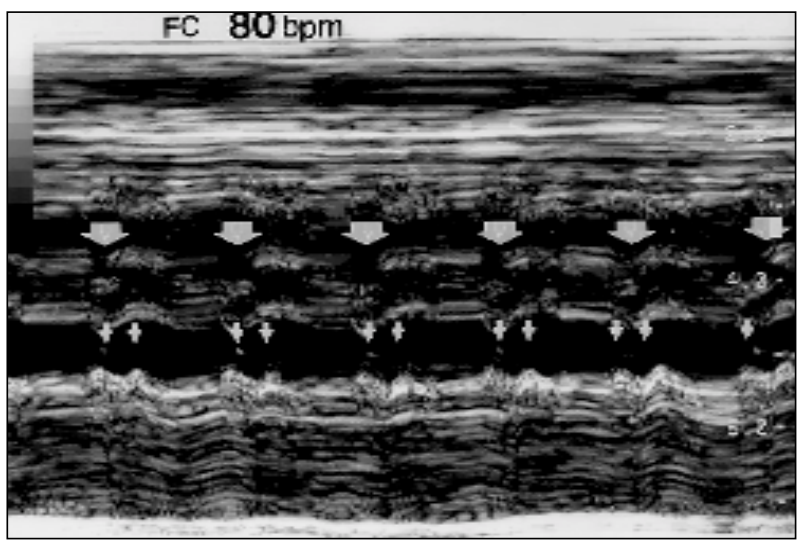

Fig. 1 - Bigeminismo atrial não conduzido em traçado de modo-M. As setas brancas finas indicam a contração da parede atrial e brancas grossas a parede ventricular cuja freqüência é bradicárdica (80bpm).

tada (paroxística) ou sustentada (contínua) ${ }^{9}$ e pode causar insuficiência cardíaca (IC) fetal, hidropisia e morte súbita ${ }^{10,11}$. Estudos com Doppler demonstram diminuição do débito cardíaco durante o período de taquicardia ${ }^{11,12}$. Este tipo de arritmia deve ser encarado como "emergência" em cardiologia fetal, pois, sabe-se que, uma vez instalada a IC com hidropisia, o prognóstico é muito pobre ${ }^{13,14}$. Consequientemente, o tratamento com antiarrítmicos deve ser introduzido rapidamente, quer seja transplacentário ou através de cordocentese ${ }^{4,9,15-19}$. O esquema mais empregado é o que utiliza digoxina, baseando-se nas doses de ataque para crianças e adultos ${ }^{9,20-22}$.

A digoxinemia materna deve ser monitorizada a cada dois dias quando se está na fase de dose de ataque, sabendo-se que a dose terapêutica fetal é atingida quando a digoxinemia materna atinge 1,5-2,0ng/mL. A possibilidade de intoxicação digitálica materna é real, sendo aconselhável que se faça todo este tratamento com monitorização materna em ambiente hospitalar. Na experiência dos autores, a porcentagem de reversão da taquicardia e evolução fetal favorável é de 93\%. Apenas dois casos apresentaram evolu-

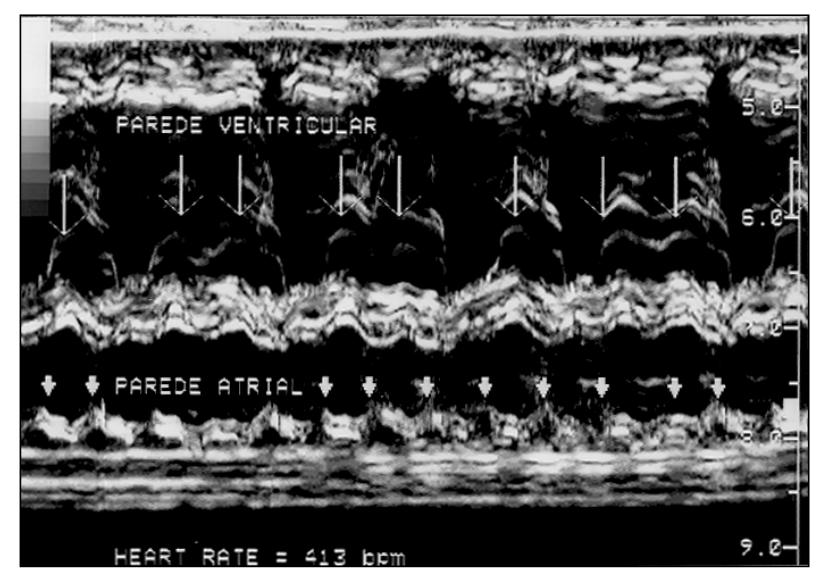

Fig. 2 - Flutter atrial. Superior: as setas longas demonstram a parede ventricular em frequiência taquicárdica porém irregular devido ao bloqueio atrioventricular variável ao nível de nó atrioventricular; inferior: as setas curtas demonstram a parede atrial em freqüência de $413 \mathrm{bpm}$. ção desfavorável seguida de óbito, atribuindo-se à hidropisia importante, já instalada por ocasião da referência da gestante ${ }^{9}$.

Flutter e fibrilação atrial - FC atrial ao modo-M entre 300-460bpm. A condução para o ventrículo é sempre acompanhada de BAV variável com conseqüente FC ventricular irregular e em torno de 200bpm (fig. 2). Pode também levar à IC e hidropisia e a terapêutica fetal deve ser instituída rapidamente ${ }^{5,9}$. O esquema terapêutico é similar ao da taquicardia supraventricular e a droga de escolha é também a digoxina.

Arritmias ventriculares - Extra-sístole ventricular O diagnóstico é feito através da demonstração do batimento precoce seguido de pausa compensatória ao modoM, passando o cursor pela parede ventricular. São bem mais raras que as extra-sístoles atriais mas, igualmente, benignas. A causa é desconhecida e não há indicação de terapêutica fetal (fig. 3).

Taquicardia ventricular - O diagnóstico é feito através da demonstração de três ou mais extra-sístoles ventriculares consecutivas, uma arritmia raramente observada em vida fetal, existindo na literatura apenas alguns relatos de caso $^{22,24}$.

Bloqueio atrioventricular - Convencionalmente, os $\mathrm{BAV}$ são divididos em $1^{\circ}, 2^{\circ}$ e $3^{\circ}$ grau. $\mathrm{OBAV}$ de $1^{\circ}$ grau além de ser raramente observado em fetos é de difícil comprovação pela impossibilidade técnica de se medir o intervalo P-R ao ecocardiograma que, no caso, encontra-se aumentado. $\mathrm{O}$ BAV de $2^{\circ}$ grau tem sido documentado com facilidade e, algumas vezes, representa estágio evolutivo de uma colagenose materna que termina por provocar o BAV de $3^{\circ}$ grau ou total $^{25,26}$.

Maior atenção tem sido dada ao BAV total, pois é o mais freqüente na vida fetal (fig. 4). Pode ocorrer isoladamente ou associado à cardiopatia. Quando isolado, a mãe freqüentemente é portadora de doença do tecido conectivo, clínica ou laboratorial, havendo presença de anticorpos anti-RO, que atravessam a placenta e podem causar lesão no sistema de condução fetal. Quando associado à cardiopatia, o prognóstico é muito pobre, especialmente se existe hidropisia ou síndrome de isomerismo atrial esquerdo ${ }^{25,27,28}$.

Nenhum esquema terapêutico eficaz tem sido utilizado para tratar o BAV total de baixa freqüência, responsável por IC e hidropisia. Poucos trabalhos relatam o uso de simpaticomiméticos para aumentar a FC em fetos hidrópicos e sem maturidade pulmonar ${ }^{28,29}$. A utilização de marcapasso diretamente no feto ou a estimulação transabdominal aplicada no abdome materno, não tem sido bem sucedida, embora seja um campo onde se desenvolvem muitas pesquisas e, provavelmente, com um futuro promissor ${ }^{20}$.

\section{Incidência}

Na experiência dos autores, a incidência dos vários tipos de arritmias fetais encontradas difere do espectro de 


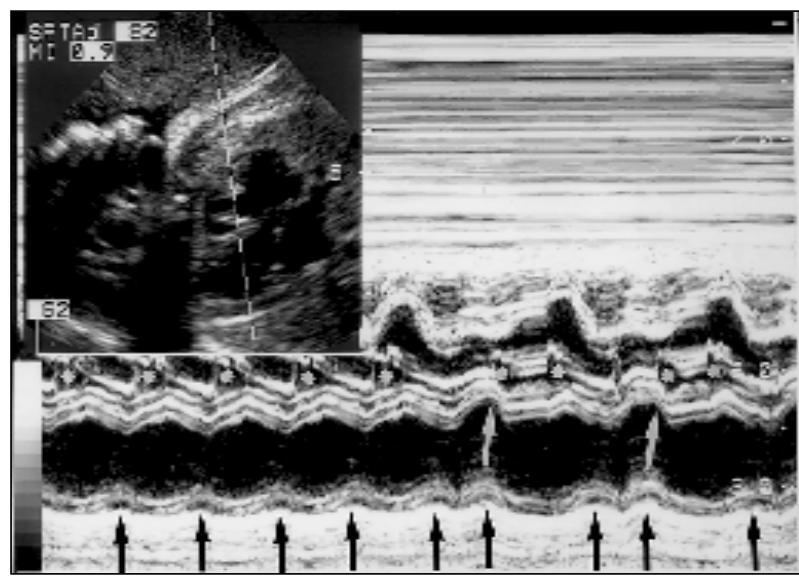

Fig. 3 - Extra-sístoles ventriculares. As setas pretas indicam a contração da parede ventricular, as brancas as extra-sístoles ventriculares com condução retrógrada para átrio e as estrelas a contração da parede atrial.

anormalidades encontradas no neonato e na criança. No período de julho/87 a abril/97 foram estudadas pela ECO fetal 3200 gestantes entre 11 a 42 semanas, tanto pela técnica transabdominal como pela técnica transvaginal. Desse total, as arritmias foram a segunda mais freqüente razão para indicação do exame (12,7\%) (tab. I). A extra-sistolia atrial foi o tipo mais freqüentemente encontrado (62\%), tendo evolução benigna e reversão em todos os casos exceto em um, que evoluiu para ritmo atrial caótico e encontra-se em acompanhamento.

Dentre os 47 fetos que se apresentaram com taquiarritmia supraventricular (taquicardia supraventricular em 41 casos e flutter atrial em seis), 16 foram do tipo não sustentada e 31 do tipo sustentada. O tratamento antiarrítmico nos últimos 35 casos, consistiu em protocolo: para o grupo não sustentada, dose de ataque de digoxina por via transplacentária (1mg VO por três dias), seguida de dose de manutenção até o parto $(0,25-0,75 \mathrm{mg}$ VO por dia); e para o grupo sustentada, dose de ataque de digoxina combinando-se as vias transplacentária (1mg VO por três dias) e direta em veia umbilical fetal $(0,04 \mathrm{mg}$ de deslanósido por kg de peso fetal

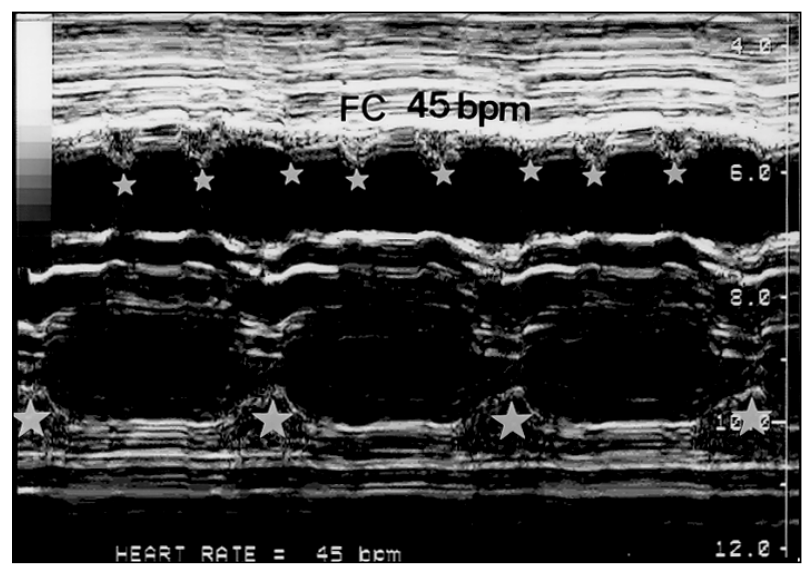

Fig. 4 - Bloqueio atrioventricular total. Notar contração atrial acima (estrelas pequenas) e a contração ventricular abaixo (estrelas grandes), com freqüência ventricular de $45 \mathrm{bpm}$. somado ao placentário), seguida de dose de manutenção até o parto $(0,25-0,75 \mathrm{mg}$ VO por dia). Os resultados mostraram que a ECO fetal foi capaz de diagnosticar, corretamente, os tipos de taquiarritmia, permitindo uma classificação tecnicamente simples, em não sustentada e sustentada, orientando a conduta terapêutica de forma adequada ao separar os grupos de acordo com a gravidade. O tempo de conversão da taquiarritmia para ritmo sinusal normal foi menor quando comparado com a literatura e o tratamento foi efetivo em $88 \%$ dos casos. A evolução perinatal deste grupo mostra que $80,7 \%$ das crianças apresentam-se vivas e bem e que existe uma tendência à normalização do ritmo cardíaco com o passar do tempo ${ }^{9}$.

Dentre os 41 casos de BAV, 32 foram do tipo total e nove de $2^{\circ}$ grau. Clinicamente dividimos estes fetos em dois grupos: 1) bloqueio associado com cardiopatia congênita complexa (1 caso) e síndrome do isomerismo atrial esquerdo (22 casos); 2) bloqueio em coração anatomicamente normal (19 casos). Dezoito óbitos ocorreram no $1^{\circ}$ grupo e apenas três no $2^{\circ}$. A associação do $2^{\circ}$ grupo com doença materna do tecido conectivo e presença de anticorpos anti-RO em sangue materno foi comprovada em $50 \%$ de nossos casos.

A administração direta em veia umbilical fetal de isoproterenol por cordocentese, foi tentada em três casos, como medida salvadora, dado o grau avançado de hidropisia fetal e freqüência ventricular extremamente baixa (entre 22 a 40bpm). Embora a FC tenha se elevado em $90 \%$ a $100 \%$ imediatamente após a cordocentese, esse aumento na freqüência ventricular não foi associado com diminuição dos edemas em nossos fetos ${ }^{29}$.

Em todos os casos de BAV indicou-se parto operatório, em razão da impossibilidade de se monitorizar o trabalho de parto.

A tabela I demonstra formas raras e complexas de arritmias, que provavelmente foram triadas para nosso Centro de Referência em Medicina e Cardiologia Fetal, por serem mais sintomáticas. Por outro lado, sabe-se que muitas destas arritmias, se não tratadas, causam hidropisia, morte súbita e óbito fetal, sendo provavelmente esta a razão da sua baixa incidência nos berçários.

\begin{tabular}{|lr|}
\hline \multicolumn{2}{|c|}{ Tabela I - Alterações de ritmo } \\
\hline Extra-sístole atrial & 160 \\
Taquicardia supraventricular & 41 \\
Flutter atrial & 6 \\
Taquicardia ventricular & 1 \\
Bloqueio atrioventricular & 32 \\
Bloqueio atrioventricular de $2^{\circ}$ Grau & 9 \\
Bigeminismo atrial bloqueado & 10 \\
Trigeminismo atrial bloqueado & 1 \\
Bigeminismo ventricular & 1 \\
Bradicardia sinusal & 2 \\
\hline Total & 263 \\
\hline
\end{tabular}




\section{Referências}

1. Allan LD, Anderson RH, Sullivan ID, Campbell S, Holt DW, Tynan M- Evaluation of fetal arrhythmias by echocardiography. Br Heart J 1983; 50: 240-5.

2. Araujo LML, Kahhale S, Faccioli R, Barbato A, Magalhães A, Zugaib M Reconhecimento das arritmias fetais pela ecodopplercardiografia. J Bras Ginec 1989; 99: 425-8.

3. Hallak M,Neerhof MG, Perry R, Nazir M, Huhta JC-Fetal supraventricular tachycardia and hydrops fetalis: combined intensive, direct, and transplacental therapy. Obstet Gynecol 1991; 78: 523-5.

4. Kleinman CS, Donnerstein RL, Jaffe CC et al - Fetal echocardiography. A tool for evaluation of in utero cardiac arrhythmias and monitoring of in utero therapy: analysis of 71 patients. Am J Cardiol 1983; 51: 237-43.

5. Kleinman CS, Copel JA - Fetal cardiac arrhythmias: diagnosis and therapy. In: Creasy RK, Resnik R., ed - Maternal-Fetal Medicine: Principles and Practice. $3^{\text {rd }}$ ed. 1994.

6. Shenker L- Fetal cardiac arrhythmias. Obstet Gynecol Survey 1979; 34:561-72.

7. Church SC, Morgan BC, Oliver Jr TK, Guntheroth WG-Cardiac arrhythmias in premature infants: an indication of autonomic immaturity? J Pediatr 1967; 71: 542-6.

8. Wheeler T, Murrills A-Patterns of fetal heart rate during normal pregnancy. Br J Obstet Gynaecol 1978; 85: 18-27.

9. Lopes LM - Taquiarritmias Fetais. Tese doutorado. Faculdade de Medicina da USP, 1995.

10. Bergmans MGM, Jonker GJ, Kock HCLV - Fetal supraventricular tachycardia. Review of the literature. Obstet Gynecol Survey 1985; 40: 61-8.

11. Kleinman CS, Donnerstein RL, Devore GR et al - Fetal echocardiography for evaluation of in utero congestive heart failure. NEngl J Med 1982; 306: 568-75.

12. Reed KL, Meijboom EJ, Sahn DJ, Scagnelli AS, Valdes-Cruz LM, Shenker L Cardiac Doppler flow velocities in human fetuses. Circulation 1986; 73: 41-6.

13. Mahony BS, Filly RA, Callen PW, Chinn DH, Golbus MS - Severe nonimmune hydrops fetalis: sonographic evaluation. Radiology 1984; 151: 757-61.

14. Stevens DC, Hilliard JK, Schreiner RL et al - Supraventricular tachycardia with edema, ascites, and hydrops in fetal sheep. Am J Obstet Gynecol 1982; 142:31622.

15. Gembruch U, Hansmann M, Redel DA, Bald R - Intrauterine therapy of fetal tachyarrhythmias: intraperitoneal administration of antiarrhythmic drugs to the fetus in fetal tachyarrhythmias with severe hydrops fetalis. J Perinat Med 1988; 16: 39-44.

16. Gembruch U, Hansmann M, Bald R - Direct intrauterine fetal treatment of fetal tachyarrhythmia with severe hydrops fetalis by antiarrhythmic drugs. Fetal Ther $1988 ; 3: 210-5$

17. Hansmann M, GembruchU, Bald R, Manz M, RedelDA-Fetal tachyarrhythmias: transplacental and direct treatment of the fetus - a report of 60 cases. Ultrasound Obstet Gynecol 1991; 1: 162-70.

18. Silverman NH, Enderlein MA, Stanger P, Teitel DF, Heymann MA, Golbus MS Recognition of fetal arrhythmias by echocardiography. J Clin Ultrasound 1985; 13: 255-63.

19. Weiner CP, Thompson MIB - Direct treatment of fetal supraventricular tachycardia after failed transplacental therapy. Am J Obstet Gynecol 1988; 158: 570-3.

20. Kleinman CS, Copel JA, Weinstein EM, Santulli Jr TV, Hobbins JC- In utero diagnosis and treatment of fetal supraventricular taquicardia. Semin Perinatol 1985; 9: 113-29.

21. Kleinman CS, Copel JA, Weinstein EM, Santulli JrTV, Hobbins JC- Treatment of fetal supraventricular tachyarrhythmias. J Clin Ultrasound 1985; 13: 265-73.

22. Smith TW, Braunwald E-Controle da insuficiência cardíaca. In: Braunwald E, ed - Tratado de Medicina Cardiovascular. São Paulo: Roca, 1987: 500-17.

23. Lopes LM, Cha SC, Scanavacca MI, Tuma-Calil VML, Zugaib M-Fetal idiopathic ventricular tachycardia with nonimmune hydrops: Benign course. Ped Cardiol 1996; 17: 192-3.

24. Sherer DM, Sadovksy E, Menashe M, Mordel N, Rein AJJT - Fetal ventricular tachycardia associated with nonimmmunologic hydrops fetalis. J Reprod Med 1990; 35: 292-4.

25. Lopes LM, Cha SC, Rumiko ER, Aiello VD, Zugaib M.- Bloqueio atrioventricular fetal. Arq Bras Cardiol 1992; 59: 261-4.

26. Lopes LM, Cha SC, Leone C, Aiello VD, Zugaib M - Experiência clínica com a ecocardiografia fetal: análise retrospectiva de 1250 casos. Rev Latin Perinat 1994; 14: 1-7.

27. Araujo LML, Silverman NH, Filly RA, Golbus MS, Finkbeiner WE, Schmidt KG - Prenatal diagnosis of left atrial isomerism by ultrasound. J Ultrasound Med 1987; 6: 667-70.

28. Schmidt KG, Ulmer HE, Silverman NH, Kleinman CS, Copel JA- Perinatal outcome of fetal complete atrioventricular block: a multicenter experience. J Am Coll Cardiol 1991; 17: 1360-6.

29. Lopes LM, Cha SC, Leone C, Zugaib M-Uso de simpaticomimético em bloqueio atrioventricular fetal. Arq Bras Cardiol 1994; 63: 297-8.

30. Walkinshaw AS, Welch CR, McCormack J, Walsh K - In utero pacing for fetal congenital heart block. Fetal Diagn Ther 1994; 9: 183-5. 